\title{
Structure, vibrational analysis and insights into host-guest interactions in as-synthesized pure silica ITQ-12 zeolite by periodic B3LYP calculations. (Supporting Information)
}

\author{
Claudio Marcelo Zicovich-Wilson, ${ }^{* a}$ María Luisa San-Román, ${ }^{b}$ \\ Miguel Angel Camblor, ${ }^{c}$ Fabien Pascale ${ }^{d}$ and José Sergio Durand-Niconoff ${ }^{\dagger a}$ \\ a Facultad de Ciencias, Universidad Autónoma del Estado de Morelos, Av. Universidad 1001, Col. \\ Chamilpa, 62209 Cuernavaca (Morelos), Mexico. \\ ${ }^{b}$ Centro Investigaciones Químicas, Universidad Autónoma del Estado de Morelos, Av. Universidad \\ 1001, Col. Chamilpa, 62209 Cuernavaca (Morelos), Mexico. \\ ${ }^{c}$ Instituto de Ciencia de Materiales de Madrid (CSIC) Departamento de Materiales Porosos y Com- \\ puestos de Intercalación C/ Sor Juana Inés de la Cruz 3 Campus Cantoblanco 28049 Madrid,Spain. \\ ${ }^{d}$ Laboratoire de Cristallographie et Modélisation des Matériaux Minéraux et Biologiques, \\ Vandoeuvre-lès-Nancy, Cedex 05, 54506 France.
}

\begin{abstract}
As-made and calcined ITQ-12 zeolites are structurally characterized by means of the analysis of their vibrational modes. The experimental IR spectra made on high crystalline samples are compared with accurate B3LYP periodic calculations performed with the CRYSTAL06 code. The fair agreement between both sets of data allows to make a reliable assignment of the IR modes. Thanks to the detailed information provided by the theoretical calculations, the analysis of the IR intensities, the Born dynamic charges, and the whole set of vibrational frequencies at $\Gamma$-point give light on several aspects of the host-guest interaction, structure-direction issues, including the role of fluoride anions in
\end{abstract}

*To whom correspondence should be addressed. E-Mail: claudio@servm.fc.uaem.mx

${ }^{\dagger}$ On leave from Universidad Veracruzana, Xalapa, Mexico 
allowing the crystallization of silica structures with strained double-four rings, and the role played by the framework flexibility.

\section{The phase transition mode of the calcined ITQ-12}

Under a detailed analysis, it turns out that the degree of agreement between calculated and experimental IR frequencies differs for each material, the calcined one exhibiting the larger deviations. This is to be attributed to the above discussed fluctuations of the framework atomic positions at room temperature that are more significant in the absence of template.

In addition, the $C 1 m 1$ monoclinic phase considered for calculation is not a stable species for the calcined material. This arises from the appearance of an imaginary frequency at $23 \mathrm{i} \mathrm{cm}^{-1}$ that corresponds to the transition between two stable triclinic phases equivalent through a mirror plane. Actually, this mode of

symmetry $A^{\prime \prime}$ is not the pure transition mode, but it is partially mixed with a translational mode of the same symmetry featuring a quasi-degenerate state, where the corresponding partner appears at $14 i \mathrm{~cm}^{-1}$.

The very low absolute value of the imaginary frequencies associated to the transition mode provides additional evidence that at normal temperatures a dynamic process should take place in the form of a fast exchange between triclinic phases. Owing to the large framework flexibility, this exchange could occur quite fast, giving rise to an average monoclinic symmetry. While the ideal $C 1 m 1$ monoclinic structure adopted for calculations is expected to be close to the average geometry of the calcined sample, the same is obviously not foreseeable as concerns frequencies, contributing to a loss in the accuracy of the simulation.

As concerns the ring units whose deformations are mainly involved in the phase transition, they are the $6 \mathrm{MR}$ and 8MR. The two former contribute equally in about $5.1 \%$ to the two quasi-degenerate imaginary modes while the contribution of $8 \mathrm{R}_{1}$ is larger than that of $8 \mathrm{R}_{2}$ with values about 5.6 and $4.8 \%$, respectively. The remaining contributions are predominantly external motions of all ring-units $(\approx 75 \%)$. 


\section{Coupling between the framework and the TMI in the soft- mode region}

In the region of soft modes most of the framework vibrations are accompanied in the as-made material by motions of the $\mathrm{TMI}^{+}$occluded. Additionally, there are some modes that are clearly attributable to the extraframework species (with contributions of more than $80 \%$ ). This is for instance as concerns the two

modes at 65 and $76 \mathrm{~cm}^{-1}$ that correspond to quasi pure roto-translational motions of the organic fragment. The framework remains practically static in the former ( $4 \%$ of total framework contribution), while in the latter a complex deformation of the $8 \mathrm{R}-\mathrm{CI}$ is exhibited, that involves a rigid rotation of the $\mathrm{D} 4 \mathrm{R}$ along the axis perpendicular to $4 \mathrm{R}_{1}$ with a deformation of $5 \mathrm{R}_{1}, 5 \mathrm{R}_{4}, 6 \mathrm{R}_{2}$ and the two $8 \mathrm{MR}$. The total framework contribution is $16 \%$, dominated by the deformation of $8 \mathrm{R}_{2}$ with $\Xi=14.5 \%$.

In most cases, those modes arising from the motion of the template inside the cavity appear associated to rotations of the methyl groups. At very low frequencies the 3-methyl is the most involved in this kind of motions, as it occurs in modes at 50 and $90 \mathrm{~cm}^{-1}$ in which together with the rotation of the 3-methyl group, the template rotate along the ring plane and around an axis parallel to the $b$ direction, respectively. The D4R unit also rotates rigidly in the former but now along the axis perpendicular to the $4 \mathrm{R}_{2}$, causing deformation of the $6 \mathrm{MR}$ and $8 \mathrm{MR}$ units. In the mode at $90 \mathrm{~cm}^{-1}$ the contribution of the framework is low and involve the deformation of all the rings. The rotation of the 3-methyl is also coupled with two of the modes that display motions of the $\mathrm{F}^{-}$inside the $\mathrm{D} 4 \mathrm{R}$ unit: at 108 and $123 \mathrm{~cm}^{-1}$ where the anion moves along the $c$ and $b$ directions, respectively. The framework contribution is low (11\%), mainly constituted by the deformation of $8 \mathrm{R}_{1}$ and $8 \mathrm{R}_{2}$ ( $\Xi=10$ and $11 \%$, respectively).

The 4- and 1-methyl groups feature rotations in modes at $159 / 175 \mathrm{~cm}^{-1}$ and $227 \mathrm{~cm}^{-1}$, respectively. As regards the modes at 159 and $227 \mathrm{~cm}^{-1}$, they are completely dominated by the 4 - and 1-methyl rotations, respectively, with practically null framework contributions. In contrast, the mode at $175 \mathrm{~cm}^{-1}$ exhibits a total framework contribution of $15 \%$, dominated by the deformations of $5 R_{2}$ and $5 R_{1}(\Xi=10$ and $9 \%$, respectively) followed by those of $8 \mathrm{R}_{1}$ and $8 \mathrm{R}_{2}$ (both with $\Xi=8 \%$ ). It is worth to recall that these rings 
are the closest ones to the 4-methyl group and the overall situation indicates stronger interactions with the framework $\mathrm{O}$ atoms than in the other two methyl groups.

\section{Analysis of the Born atomic charges}

As regards the silicon dynamic charges, they do not display a trend similar to the static ones and, actually, the largest values appear on atoms 7 and 8 that do not belong to the D4R unit. The fact that the framework is more statically than dynamically affected by the presence of the $\mathrm{F}^{-}$is to be related to its extremal spatial confinement $^{1,2}$ in the $\mathrm{D} 4 \mathrm{R}$ unit that strongly restricts its motions.

In what concerns the $\mathrm{O}$ atoms, the maximal dissimilarities in the dynamic charges occur in some of them that are close to some of the $\mathrm{TMI}^{+} \mathrm{H}$ atoms (lowest values in the bottom panel of Figure 10). The most affected are those labeled as 13, 19, 22 and 23 in Figure 10. The first and the last ones, display close contacts with the $\mathrm{H}$ atoms of the 4-methyl group of the $\mathrm{TMI}^{+}$(see Table 3 ), that in previous sections has been identified as the most acidic and the one with the strongest interaction with the framework $\mathrm{O}$ atoms.

As regards atoms 22 and 19, the former is at close distance (2.67 $\AA$ ) to the $\mathrm{H}$ atom in position 5 of the imidazolium ring, while the latter is close $(2.64 \AA)$ to an $\mathrm{H}$ atom of the 1-methyl group.

\section{References}

1. Villaescusa, L.; Márquez, F.; Zicovich-Wilson, C. M.; Camblor, M. A. J. Phys. Chem. B 2002, 106, $2796-2800$.

2. George, A. R.; Catlow, C. R. A. Chem. Phys. Lett. 1995, 247, 408-417. 
Table S1: Fractional coordinates of the asymmetric unit in the primitive cell $(a=b=9.11542935 \AA, c=$ $8.86390000 \AA, \alpha=81.365108^{\circ}, \beta=98.634892^{\circ}, \gamma=69.075737^{\circ}$ ) of the calcined material, optimized with cell fixed.

\begin{tabular}{cccc} 
Atom & $X$ & $Y$ & $Z$ \\
\hline $\mathrm{Si}$ & -0.11933 & -0.39706 & 0.12374 \\
$\mathrm{Si}$ & 0.10617 & -0.31080 & -0.31041 \\
$\mathrm{Si}$ & 0.00602 & -0.21072 & 0.33018 \\
$\mathrm{Si}$ & -0.40890 & -0.09777 & -0.08166 \\
$\mathrm{Si}$ & -0.28942 & 0.08038 & 0.35364 \\
$\mathrm{Si}$ & -0.19082 & -0.01717 & -0.29114 \\
$\mathrm{O}$ & 0.00588 & -0.33200 & 0.21431 \\
$\mathrm{O}$ & -0.17918 & -0.09660 & 0.32936 \\
$\mathrm{O}$ & 0.09980 & -0.31693 & -0.49390 \\
$\mathrm{O}$ & -0.04287 & -0.46853 & -0.01604 \\
$\mathrm{O}$ & 0.23753 & -0.23753 & -0.24919 \\
$\mathrm{O}$ & -0.29560 & -0.25260 & 0.05728 \\
$\mathrm{O}$ & 0.15964 & -0.49189 & -0.20853 \\
$\mathrm{O}$ & 0.09988 & -0.09988 & 0.26791 \\
$\mathrm{O}$ & -0.46715 & 0.14656 & 0.24318 \\
$\mathrm{O}$ & -0.08466 & 0.08466 & -0.24205 \\
$\mathrm{O}$ & -0.29916 & -0.01858 & -0.16354 \\
$\mathrm{O}$ & -0.07198 & -0.19985 & -0.29042 \\
$\mathrm{O}$ & -0.30697 & 0.06778 & -0.46491 \\
$\mathrm{O}$ & -0.20665 & 0.20665 & 0.30706 \\
\hline
\end{tabular}


Table S2: Fractional coordinates in the primitive cell $(a=9.20883683 \AA, b=9.17455122 \AA, c=8.98437451$ $\AA, \alpha=81.022703^{\circ}, \beta=98.201409^{\circ}, \gamma=68.486083^{\circ}$ ) of the calcined material, fully optimized.

\begin{tabular}{cccc||cccc} 
Atom & $X$ & $Y$ & $Z$ & Atom & $X$ & $Y$ & $Z$ \\
\hline $\mathrm{Si}$ & -0.10883 & -0.39205 & 0.11926 & $\mathrm{O}$ & -0.03243 & -0.47351 & -0.01400 \\
$\mathrm{Si}$ & 0.40452 & 0.12182 & 0.11813 & $\mathrm{O}$ & 0.47487 & 0.03544 & -0.01512 \\
$\mathrm{Si}$ & 0.09905 & -0.30892 & -0.32374 & $\mathrm{O}$ & 0.23609 & -0.24565 & -0.26336 \\
$\mathrm{Si}$ & 0.30653 & -0.10965 & -0.31008 & $\mathrm{O}$ & -0.28536 & -0.25212 & 0.04398 \\
$\mathrm{Si}$ & 0.01208 & -0.20149 & 0.31921 & $\mathrm{O}$ & 0.25732 & 0.29381 & 0.03976 \\
$\mathrm{Si}$ & 0.21957 & -0.00161 & 0.33304 & $\mathrm{O}$ & 0.14587 & -0.48983 & -0.22520 \\
$\mathrm{Si}$ & -0.41046 & -0.10024 & -0.09048 & $\mathrm{O}$ & 0.48272 & -0.16558 & -0.20140 \\
$\mathrm{Si}$ & 0.09738 & 0.40678 & -0.09110 & $\mathrm{O}$ & 0.12026 & -0.10315 & 0.27053 \\
$\mathrm{Si}$ & -0.28655 & 0.09636 & 0.33768 & $\mathrm{O}$ & -0.46101 & 0.15908 & 0.22476 \\
$\mathrm{Si}$ & -0.07699 & 0.29827 & 0.35206 & $\mathrm{O}$ & -0.13033 & 0.47816 & 0.24988 \\
$\mathrm{Si}$ & -0.20019 & -0.00921 & -0.30710 & $\mathrm{O}$ & -0.10377 & 0.09690 & -0.25067 \\
$\mathrm{Si}$ & 0.00905 & 0.19152 & -0.29308 & $\mathrm{O}$ & -0.31821 & -0.01547 & -0.19178 \\
$\mathrm{O}$ & 0.00881 & -0.31585 & 0.19899 & $\mathrm{O}$ & 0.01446 & 0.29862 & -0.16688 \\
$\mathrm{O}$ & 0.34411 & 0.00364 & 0.22384 & $\mathrm{O}$ & -0.07436 & -0.19077 & -0.30092 \\
$\mathrm{O}$ & -0.17041 & -0.07595 & 0.30978 & $\mathrm{O}$ & 0.18936 & 0.06306 & -0.28465 \\
$\mathrm{O}$ & 0.09635 & 0.18146 & 0.32714 & $\mathrm{O}$ & -0.30524 & 0.07489 & -0.48306 \\
$\mathrm{O}$ & 0.08996 & -0.31234 & 0.49420 & $\mathrm{O}$ & -0.06465 & 0.30684 & -0.46661 \\
$\mathrm{O}$ & 0.32003 & -0.09123 & -0.49064 & $\mathrm{O}$ & -0.21108 & 0.22934 & 0.29836 \\
\hline
\end{tabular}


Table S3: Fractional coordinates in the primitive cell $(a=9.13399000 \AA, b=9.13399000 \AA, c=8.83660000$ $\AA, \alpha=80.966120^{\circ}, \beta=99.033880^{\circ}, \gamma=69.768310^{\circ}$ ) of the as-made material, optimized with cell fixed.

\begin{tabular}{cccc||cccc} 
Atom & $X$ & $Y$ & $Z$ & Atom & $X$ & $Y$ & $Z$ \\
\hline $\mathrm{Si}$ & -0.11952 & -0.40310 & 0.11582 & $\mathrm{O}$ & -0.09295 & 0.11947 & -0.24332 \\
$\mathrm{Si}$ & 0.39570 & 0.10876 & 0.12174 & $\mathrm{O}$ & -0.28663 & -0.00336 & -0.15895 \\
$\mathrm{Si}$ & 0.11465 & -0.29194 & -0.30469 & $\mathrm{O}$ & 0.02409 & 0.33134 & -0.20133 \\
$\mathrm{Si}$ & 0.31047 & -0.08206 & -0.32880 & $\mathrm{O}$ & -0.04785 & -0.19116 & -0.25229 \\
$\mathrm{Si}$ & -0.00309 & -0.21385 & 0.33715 & $\mathrm{O}$ & 0.20606 & 0.08671 & -0.28975 \\
$\mathrm{Si}$ & 0.19522 & -0.00499 & 0.31320 & $\mathrm{O}$ & -0.29856 & 0.05323 & -0.45879 \\
$\mathrm{Si}$ & -0.39924 & -0.08815 & -0.09110 & $\mathrm{O}$ & -0.06191 & 0.31213 & -0.49122 \\
$\mathrm{Si}$ & 0.11077 & 0.41571 & -0.08959 & $\mathrm{O}$ & -0.23790 & 0.21767 & 0.29003 \\
$\mathrm{Si}$ & -0.29151 & 0.07169 & 0.35555 & $\mathrm{~F}$ & 0.00899 & -0.00778 & -0.48585 \\
$\mathrm{Si}$ & -0.09364 & 0.28211 & 0.33070 & $\mathrm{~N}$ & -0.39587 & -0.41592 & -0.47190 \\
$\mathrm{Si}$ & -0.17431 & -0.00588 & -0.28583 & $\mathrm{C}$ & -0.46822 & 0.48834 & -0.41679 \\
$\mathrm{Si}$ & 0.01999 & 0.20524 & -0.31140 & $\mathrm{~N}$ & 0.43996 & 0.46777 & 0.45888 \\
$\mathrm{O}$ & -0.00321 & -0.34530 & 0.23036 & $\mathrm{C}$ & 0.45456 & -0.44587 & 0.31939 \\
$\mathrm{O}$ & 0.31047 & -0.00952 & 0.18773 & $\mathrm{C}$ & -0.44298 & -0.37337 & 0.36541 \\
$\mathrm{O}$ & -0.19159 & -0.09818 & 0.31382 & $\mathrm{C}$ & -0.28160 & -0.36456 & -0.38110 \\
$\mathrm{O}$ & 0.07203 & 0.18177 & 0.28265 & $\mathrm{C}$ & 0.34452 & 0.36690 & 0.47304 \\
$\mathrm{O}$ & 0.07906 & -0.32027 & -0.48302 & $\mathrm{C}$ & 0.37566 & -0.44001 & 0.15702 \\
$\mathrm{O}$ & 0.31889 & -0.06691 & 0.48648 & $\mathrm{H}$ & -0.16663 & -0.41458 & -0.41187 \\
$\mathrm{O}$ & -0.02669 & -0.47271 & -0.01495 & $\mathrm{H}$ & -0.32677 & -0.23448 & -0.40971 \\
$\mathrm{O}$ & 0.48544 & 0.03742 & -0.00939 & $\mathrm{H}$ & -0.26867 & -0.40769 & -0.25614 \\
$\mathrm{O}$ & 0.26275 & -0.23293 & -0.26311 & $\mathrm{H}$ & 0.42032 & 0.24221 & 0.48434 \\
$\mathrm{O}$ & -0.29093 & -0.25708 & 0.03462 & $\mathrm{H}$ & 0.29295 & 0.38154 & -0.42460 \\
$\mathrm{O}$ & 0.26081 & 0.28751 & 0.04658 & $\mathrm{H}$ & 0.24762 & 0.40747 & 0.36865 \\
$\mathrm{O}$ & 0.18328 & -0.47369 & -0.19279 & $\mathrm{H}$ & 0.41991 & 0.44258 & 0.12893 \\
$\mathrm{O}$ & 0.49423 & -0.12969 & -0.23110 & $\mathrm{H}$ & 0.24571 & -0.39909 & 0.14050 \\
$\mathrm{O}$ & 0.10653 & -0.12444 & 0.26731 & $\mathrm{H}$ & 0.40186 & -0.35620 & 0.07361 \\
$\mathrm{O}$ & -0.47810 & 0.12521 & 0.26316 & $\mathrm{H}$ & -0.45640 & 0.43737 & -0.29508 \\
$\mathrm{O}$ & -0.16429 & 0.46063 & 0.21527 & $\mathrm{H}$ & -0.40027 & -0.29685 & 0.29556 \\
\hline & & & & & & & \\
\hline & & & & & &
\end{tabular}


Table S4: Fractional coordinates in the primitive cell $(a=9.25175553 \AA, b=9.21848208 \AA, c=8.92388254$ $\AA, \alpha=80.418404^{\circ}, \beta=98.534010^{\circ}, \gamma=69.765180^{\circ}$ ) of the as-made material, fully optimized.

\begin{tabular}{cccc||cccc} 
Atom & $X$ & $Y$ & $Z$ & Atom & $X$ & $Y$ & $Z$ \\
\hline $\mathrm{Si}$ & -0.08951 & -0.40573 & 0.12484 & $\mathrm{O}$ & -0.11603 & 0.11449 & -0.24080 \\
$\mathrm{Si}$ & 0.42027 & 0.10306 & 0.12550 & $\mathrm{O}$ & -0.32097 & -0.00825 & -0.18763 \\
$\mathrm{Si}$ & 0.09187 & -0.29437 & -0.31548 & $\mathrm{O}$ & 0.01140 & 0.30895 & -0.16655 \\
$\mathrm{Si}$ & 0.29754 & -0.09587 & -0.30345 & $\mathrm{O}$ & -0.06777 & -0.19270 & -0.26161 \\
$\mathrm{Si}$ & 0.00157 & -0.18991 & 0.32669 & $\mathrm{O}$ & 0.19367 & 0.06207 & -0.24970 \\
$\mathrm{Si}$ & 0.20931 & 0.00800 & 0.33872 & $\mathrm{O}$ & -0.30240 & 0.05847 & -0.47466 \\
$\mathrm{Si}$ & -0.39425 & -0.11628 & -0.08512 & $\mathrm{O}$ & -0.04646 & 0.30637 & -0.46113 \\
$\mathrm{Si}$ & 0.11894 & 0.39516 & -0.08708 & $\mathrm{O}$ & -0.22576 & 0.24442 & 0.30426 \\
$\mathrm{Si}$ & -0.28282 & 0.09614 & 0.34495 & $\mathrm{~F}$ & 0.00684 & -0.00256 & -0.47566 \\
$\mathrm{Si}$ & -0.07644 & 0.29535 & 0.35631 & $\mathrm{~N}$ & -0.40736 & -0.41714 & -0.48050 \\
$\mathrm{Si}$ & -0.19316 & -0.00774 & -0.29670 & $\mathrm{C}$ & -0.48079 & 0.48681 & -0.43217 \\
$\mathrm{Si}$ & 0.01168 & 0.19077 & -0.28527 & $\mathrm{~N}$ & 0.43207 & 0.47052 & 0.44061 \\
$\mathrm{O}$ & 0.00141 & -0.30573 & 0.20559 & $\mathrm{C}$ & 0.45112 & -0.44063 & 0.30613 \\
$\mathrm{O}$ & 0.33324 & 0.01024 & 0.22655 & $\mathrm{C}$ & -0.44878 & -0.37050 & 0.35790 \\
$\mathrm{O}$ & -0.18082 & -0.06316 & 0.29220 & $\mathrm{C}$ & -0.29727 & -0.36927 & -0.38360 \\
$\mathrm{O}$ & 0.08494 & 0.19210 & 0.30719 & $\mathrm{C}$ & 0.33492 & 0.37283 & 0.44694 \\
$\mathrm{O}$ & 0.05948 & -0.30625 & -0.49783 & $\mathrm{C}$ & 0.37653 & -0.43037 & 0.14321 \\
$\mathrm{O}$ & 0.31783 & -0.05862 & -0.48334 & $\mathrm{H}$ & -0.18118 & -0.41862 & -0.40968 \\
$\mathrm{O}$ & 0.02773 & 0.48807 & 0.03162 & $\mathrm{H}$ & -0.34118 & -0.23999 & -0.41023 \\
$\mathrm{O}$ & -0.47633 & -0.02054 & 0.03606 & $\mathrm{H}$ & -0.29046 & -0.41412 & -0.26098 \\
$\mathrm{O}$ & 0.24362 & -0.24688 & -0.26366 & $\mathrm{H}$ & 0.41115 & 0.24853 & 0.49776 \\
$\mathrm{O}$ & -0.25747 & -0.28766 & 0.00869 & $\mathrm{H}$ & 0.24729 & 0.41584 & -0.48491 \\
$\mathrm{O}$ & 0.29320 & 0.26495 & 0.00471 & $\mathrm{H}$ & 0.27459 & 0.38416 & 0.32893 \\
$\mathrm{O}$ & 0.14762 & -0.47974 & -0.21940 & $\mathrm{H}$ & 0.40784 & 0.45180 & 0.11847 \\
$\mathrm{O}$ & 0.47659 & -0.15858 & -0.19544 & $\mathrm{H}$ & 0.24792 & -0.37298 & 0.12053 \\
$\mathrm{O}$ & 0.12742 & -0.11102 & 0.28299 & $\mathrm{H}$ & 0.41837 & -0.35967 & 0.06267 \\
$\mathrm{O}$ & -0.46281 & 0.15789 & 0.23843 & $\mathrm{H}$ & -0.47214 & 0.43225 & -0.31314 \\
$\mathrm{O}$ & -0.12889 & 0.47881 & 0.25752 & $\mathrm{H}$ & -0.40363 & -0.29368 & 0.29306 \\
\hline & & & & & & & \\
\hline
\end{tabular}


Table S5: Calculated frequencies $\left(\mathrm{cm}^{-1}\right)$ and IR intensities $\left(\mathrm{km} \mathrm{mol}^{-1}\right)$ of the calcined material

\begin{tabular}{|c|c|c|c|c|c|c|c|c|c|c|c|}
\hline Freq & Intens & Freq & Intens & Freq & Intens & Freq & Intens & Freq & Intens & Freq & Intens \\
\hline-13.9045 & 0.01 & -9.2881 & 0.00 & 18.4854 & 0.10 & 41.2800 & 0.38 & 67.2339 & 0.51 & 89.9357 & 0.55 \\
\hline 97.3642 & 0.71 & 111.1826 & 1.08 & 111.8564 & 1.81 & 119.2654 & 0.27 & 131.8674 & 0.81 & 133.5645 & 0.14 \\
\hline 138.3935 & 2.99 & 155.9308 & 0.82 & 159.1262 & 1.52 & 169.0569 & 2.22 & 181.4901 & 4.37 & 191.9572 & 0.08 \\
\hline 197.3197 & 0.85 & 198.6553 & 3.06 & 210.7825 & 0.78 & 225.9717 & 7.67 & 232.3856 & 1.70 & 240.6979 & 0.03 \\
\hline 243.8349 & 26.10 & 251.8892 & 7.55 & 257.4864 & 1.00 & 274.9860 & 86.26 & 278.9523 & 13.98 & 281.8894 & 4.06 \\
\hline 289.5870 & 21.95 & 294.9258 & 23.98 & 304.8155 & 13.22 & 309.9005 & 0.03 & 315.5357 & 46.99 & 328.1764 & 0.13 \\
\hline 335.8032 & 72.41 & 335.9959 & 4.48 & 351.9979 & 2.03 & 359.4110 & 3.49 & 361.6589 & 446.43 & 370.9303 & 89.13 \\
\hline 377.1906 & 158.96 & 387.5181 & 82.19 & 398.4419 & 88.20 & 400.6655 & 73.63 & 406.0476 & 138.29 & 408.0005 & 16.89 \\
\hline 414.9569 & 263.31 & 417.6196 & 224.72 & 423.0518 & 94.26 & 427.6707 & 215.92 & 429.5473 & 158.14 & 431.2597 & 407.97 \\
\hline 436.3913 & 135.39 & 439.7910 & 682.84 & 459.3920 & 288.50 & 462.5961 & 557.26 & 467.5578 & 63.55 & 501.1114 & 8.25 \\
\hline 563.9146 & 283.36 & 567.7602 & 24.09 & 589.3358 & 155.66 & 590.9056 & 58.41 & 594.9609 & 25.21 & 614.0140 & 852.39 \\
\hline 652.7397 & 0.55 & 678.9276 & 1.51 & 719.8289 & 3.27 & 747.9962 & 106.58 & 785.2425 & 0.93 & 798.4588 & 5.91 \\
\hline 802.0590 & 7.45 & 806.5786 & 87.56 & 814.9855 & 60.07 & 817.3296 & 115.72 & 822.0929 & 35.01 & 823.5902 & 7.95 \\
\hline 831.4959 & 13.86 & 832.3914 & 2.76 & 834.6159 & 48.52 & 846.6396 & 351.40 & 850.8213 & 6.55 & 1074.1029 & 8.45 \\
\hline 1077.7567 & 176.41 & 1084.0728 & 251.33 & 1086.4991 & 19.96 & 1092.4842 & 876.92 & 1096.8737 & 5690.69 & 1101.6637 & 1749.90 \\
\hline 1103.1852 & 4693.09 & 1104.3712 & 145.72 & 1114.5641 & 2105.55 & 1125.1357 & 4108.86 & 1128.0963 & 31.27 & 1131.1197 & 789.36 \\
\hline 1155.4716 & 8.72 & 1188.1894 & 21.50 & 1191.1530 & 18.06 & 1208.3077 & 7.46 & 1210.4057 & 71.49 & 1214.0233 & 262.65 \\
\hline 1221.2784 & 150.80 & 1238.5696 & 59.32 & 1248.1825 & 25.98 & 1255.1422 & 9.44 & 1259.0581 & 23.73 & & \\
\hline
\end{tabular}

Table S6: Calculated frequencies $\left(\mathrm{cm}^{-1}\right)$ and IR intensities $\left(\mathrm{km} \mathrm{mol}^{-1}\right)$ of the as-made material.

\begin{tabular}{|c|c|c|c|c|c|c|c|c|c|c|c|}
\hline Freq & Intens & Freq & Intens & Freq & Intens & Freq & Intens & Freq & Intens & Freq & Intens \\
\hline 116.5740 & 40.06 & 122.8132 & 60.37 & 127.3942 & 67.37 & 129.3148 & 88.45 & 130.4847 & 19.67 & 137.4167 & 148.70 \\
\hline 142.4354 & 146.08 & 156.7523 & 57.54 & 158.9109 & 4.93 & 166.3838 & 3.31 & 167.0828 & 3.15 & 174.9091 & 9.41 \\
\hline 217.0487 & 8.02 & 220.1263 & 4.04 & 227.2231 & 7.72 & 234.9424 & 18.55 & 237.5401 & 26.18 & 242.2881 & 7.41 \\
\hline 247.1945 & 8.15 & 256.6202 & 16.99 & 260.5104 & 6.80 & 263.6321 & 12.40 & 266.1366 & 20.63 & 284.8468 & 9.22 \\
\hline 289.3113 & 21.06 & 290.4848 & 7.83 & 299.8204 & 6.50 & 302.7540 & 4.49 & 305.6826 & 3.80 & 325.4139 & 11.62 \\
\hline 390.2287 & 4.12 & 392.1091 & 200.19 & 398.1097 & 197.06 & 401.5712 & 124.09 & 408.8074 & 55.16 & 414.1178 & 589.03 \\
\hline 421.9552 & 504.94 & 427.3360 & 245.94 & 428.3447 & 104.98 & 430.1032 & 249.32 & 434.0365 & 67.07 & 442.8783 & 162.99 \\
\hline 443.5569 & 315.12 & 482.6695 & 89.85 & 488.5474 & 70.31 & 491.2259 & 49.24 & 497.5889 & 23.57 & 505.7359 & 11.53 \\
\hline 571.9671 & 9.12 & 574.2979 & 48.26 & 580.3589 & 161.14 & 580.8391 & 266.98 & 595.6162 & 410.72 & 604.9773 & 1342.12 \\
\hline 613.6441 & 25.81 & 633.9315 & 56.45 & 646.3516 & 2.78 & 666.7274 & 1.43 & 671.2178 & 7.95 & 692.6146 & 6.62 \\
\hline 709.8041 & 0.25 & 715.8189 & 168.62 & 758.1884 & 10.68 & 767.3768 & 6.59 & 774.0908 & 5.52 & 784.4448 & 236.99 \\
\hline 786.1819 & 43.49 & 794.4124 & 34.62 & 798.2035 & 66.29 & 798.6514 & 43.21 & 807.7024 & 119.69 & 812.2839 & 96.87 \\
\hline 1137.4929 & 17.20 & 1167.3960 & 115.34 & 1174.5673 & 174.55 & 1182.1154 & 128.93 & 1185.5137 & 350.00 & 1189.7831 & 42.63 \\
\hline 1192.0254 & 66.26 & 1195.3299 & 16.16 & 1202.0638 & 68.30 & 1210.7745 & 7.84 & 1215.3722 & 227.13 & 1221.7535 & 53.00 \\
\hline 1230.7236 & 39.67 & 1232.1077 & 0.82 & 1245.1840 & 99.95 & 1290.2769 & 12.61 & 1379.2373 & 0.31 & 1403.7684 & 1.58 \\
\hline 1447.3308 & 10.66 & 1480.1498 & 28.82 & 1489.0823 & 2.95 & 1525.8935 & 18.38 & 1529.6819 & 33.77 & 1530.8129 & 31.05 \\
\hline 1532.8435 & 5.54 & 1542.0298 & 31.78 & 1550.6706 & 40.80 & 1552.1290 & 20.25 & 1607.5471 & 115.59 & 1672.7496 & 34.16 \\
\hline 3096.4535 & 5.61 & 3121.4735 & 8.06 & 3129.7938 & 5.07 & 3175.1753 & 1.66 & 3182.1005 & 0.48 & 3212.4622 & 1.08 \\
\hline 3227.7364 & 0.54 & 3235.1067 & 1.81 & 3241.0335 & 0.58 & 3334.5394 & 32.16 & 3369.5129 & 23.26 & & \\
\hline
\end{tabular}


Table S7: Square root of the mean quadratic deviation of the OSiO angles from perfect tetrahedron shape (in degree)

\begin{tabular}{lcc}
$\mathrm{NSi}$ & calc & as-made \\
\hline 1 & 2.34384 & 2.37849 \\
2 & 2.34384 & 1.64096 \\
3 & 0.486731 & 5.17145 \\
4 & 0.486731 & 4.06829 \\
5 & 0.743382 & 4.2691 \\
6 & 0.743382 & 4.58828 \\
7 & 1.96142 & 2.06479 \\
8 & 1.96142 & 2.49753 \\
9 & 0.914946 & 4.02783 \\
10 & 0.914946 & 4.74964 \\
11 & 1.3224 & 4.80236 \\
12 & 1.3224 & 4.10997 \\
\hline
\end{tabular}

